\title{
CD47 expression regulated by the miR-133a tumor suppressor is a novel prognostic marker in esophageal squamous cell carcinoma
}

\author{
SHIGEMASA SUZUKI $^{1}$, TAKEHIKO YOKOBORI ${ }^{1}$, NARITAKA TANAKA ${ }^{1}$, MAKOTO SAKAI $^{1}$, \\ AKIHIKO SANO $^{1}$, TAKANORI INOSE ${ }^{1}$, MAKOTO SOHDA ${ }^{1}$, MASANOBU NAKAJIMA ${ }^{2}$, \\ TATSUYA MIYAZAKI $^{1}$, HIROYUKI KATO ${ }^{2}$ and HIROYUKI KUWANO ${ }^{1}$ \\ ${ }^{1}$ Department of General Surgical Science, Gunma University, Graduate School of Medicine, Gunma 371-8511; \\ ${ }^{2}$ Department of Surgical Oncology, Dokkyo Medical University, Tochigi 321-0293, Japan
}

Received January 4, 2012; Accepted March 2, 2012

DOI: $10.3892 /$ or.2012.1831

\begin{abstract}
CD47 inhibits phagocytosis and its overexpression is correlated with poor prognosis in patients with several types of cancer. It has also been reported that $C D 47$ expression in multiple sclerosis is regulated by microRNAs. However, the regulatory mechanism of $C D 47$ in cancer tissues has not been yet clarified. Re-analysis of a public microarray database revealed that miR-133a is downregulated in esophageal squamous cell carcinoma (ESCC). Moreover, in silico algorithms predicted that miR-133a is a regulator of $C D 47$. The purpose of this study was to clarify the clinical significance of $C D 47$ and its regulatory mechanism by miR-133a in ESCC. Quantitative real-time RT-PCR was used to evaluate $C D 47$ and miR-133a expression in 102 cases of curative resected ESCC and adjacent non-cancerous tissue. The regulation of $C D 47$ by miR-133a was examined with precursor miR-133a-transfected cells. A mouse xenograft model was used to investigate the ability of miR-133a to suppress tumor progression. High expression levels of $C D 47$ were associated with lymph node metastasis $(\mathrm{P}=0.049)$. Multivariate analysis showed that $C D 47$ expression was an independent prognostic factor $(\mathrm{P}=0.045)$ miR-133a expression was significantly lower in cancer tissues compared to adjacent non-cancerous tissues $(\mathrm{P}<0.001)$. In vitro assays showed that miR-133a is a direct regulator of CD47. miR-133a significantly inhibited tumorigenesis and growth in vivo. CD47 expression is a novel prognostic marker in ESCC that is directly inhibited by the miR-133a tumor suppressor. This correlation could provide new insight into the mechanism of cancer progression and a promising candidate for target therapy in ESCC.
\end{abstract}

Correspondence to: Dr Shigemasa Suzuki, Department of General Surgical Science, Gunma University, Graduate School of Medicine, 3-39-22 Showa-machi, Maebashi, Gunma 371-8511, Japan

E-mail: suzukis@med.gunma-u.ac.jp

Key words: CD47, miR-133a, esophageal cancer

\section{Introduction}

Esophageal squamous cell carcinoma (ESCC) has one of the highest mortality rates among patients with solid tumors due to the fact that it is a highly aggressive malignancy with early lymphatic and hematogenous dissemination $(1,2)$. Recent advances in perioperative management, combined with chemotherapy or chemoradiotherapy, have led to improved survival rates. However, the prognosis for patients with advanced disease remains poor and unsatisfactory (3-5). Discovering suitable biomarkers of malignancy could enhance monitoring for cancer recurrence, and the development of new therapeutic approaches is essential for the improvement of survival rates.

CD47 is a widely expressed transmembrane protein that has been implicated in multiple cellular processes, including a function as a 'do not eat me' signal inhibiting macrophage activity by binding the signal regulatory protein $\alpha$, which is expressed on phagocytes (6-8). Whereas this function is partly attributed to 'self recognition' in normal physiological conditions, several human cancers appear to upregulate CD47 to evade the tumor immunosurveillance system (9-11). Overexpression of $C D 47$ correlates with poor prognosis in acute myeloid leukemia (12), acute lymphoblastic leukemia (13) and breast cancer (14). However, the clinicopathological and prognostic significance of CD47 expression in ESCC remains unclear.

It was reported that $C D 47$ expression in multiple sclerosis is regulated by microRNAs (miRNAs) including miR-34a, miR-155 and miR-326 (15). However, the mechanism of CD47 regulation in cancer tissues has not yet been clarified. miRNAs are small non-coding RNAs of 18-25 nucleotides that partially bind the 3' untranslated region (3'-UTR) of their target mRNA, resulting in mRNA degradation and/or translational repression (16). By downregulating their target gene expression, miRNAs play an essential role in several cellular processes, such as proliferation, differentiation and apoptosis $(17,18)$. Moreover, miRNAs also function as oncogenes or tumor suppressors depending on their targets. Therefore, miRNA has garnered attention as a new diagnostic and therapeutic tool for human malignancies $(19,20)$. 
In a search for $C D 47$ regulatory miRNAs downregulated in ESCC, we re-analyzed GSE6188, the Gene Expression Omnibus public microarray database from the National Center for Biotechnical Information (21). Five downregulated miRNAs were found in ESCC. Among them, miR-133a is downregulated in the miRNA expression signatures of several types of human malignancies relative to normal adjacent tissue: head and neck squamous cell carcinoma (22-24), bladder cancer $(25,26)$, colorectal cancer $(27)$, and rhabdomyosarcoma $(28,29)$. Transfection of miR-133a induced reductions in cell proliferation, migration and invasion (25). These data suggested that miR-133a may function as a tumor suppressor. Furthermore, the TargetScan 5.0 in silico prediction algorithm (30) also indicated that miR-133a is a conserved regulatory miRNA of $C D 47$. Based on these results, we focused on the miR-133a tumor suppressor as a direct regulator of $C D 47$.

The purpose of this study was to clarify the clinical significance and regulatory mechanism of CD47 in ESCC. Firstly, we examined the expression level of $C D 47$ in ESCC and adjacent non-cancerous tissue. Secondly, we evaluated the CD47 regulation in ESCC by miR-133a in silico and in vitro, and investigated the functional analysis of the miR-133a using a mouse xenograft model.

\section{Materials and methods}

Clinical samples and RNA isolation. The pair of primary ESCC and corresponding normal esophageal epithelia were obtained from 102 ESCC patients (89 males and 13 females), who had undergone potentially curative surgery at the Department of General Surgical Science, Gunma University, between 1990 and 2007, after obtaining their written informed consent. The patients' ages ranged from 42 to 83 years, with a mean of 64.9. The median follow-up period for survivors was 40 months (range, 1-126 months). The pathological features of the specimens were classified based on the 6th edition of the TNM classification of the International Union against Cancer (UICC). The operations were classified as curative surgeries; there was no evidence of residual tumors, and the resected margins were microscopically free of tumors (R0). Normal tissues were obtained far from the center of the cancer in surgical specimens. All specimens were immediately frozen in liquid nitrogen and stored at $-80^{\circ} \mathrm{C}$ until RNA extraction. Total RNA was extracted using the miRNeasy Mini kit (Qiagen) according to the manufacturer's instructions. The quantity of isolated RNA was measured by the ND-1000 spectrophotometer (NanoDrop Technologies).

Evaluation of CD47 expression in clinical samples. cDNA for CD47 mRNA quantitative real-time reverse transcriptase PCR (RT-PCR) was synthesized from $1 \mu \mathrm{g}$ total RNA with the Omniscript Reverse Transcriptase kit (Qiagen) in a reaction volume of $20 \mu \mathrm{l}\left(60 \mathrm{~min}\right.$ at $37^{\circ} \mathrm{C}$ and $5 \mathrm{~min}$ at $93^{\circ} \mathrm{C}$ before being put on ice). The cDNA samples were stored at $-30^{\circ} \mathrm{C}$ until needed for analyses. The sequences of the $C D 47$ primers were as follows: sense primer, 5'-GGCAATGACGAAGGA GGTTA-3'; antisense primer, 5'-ATCCGGTGGTATGGAT GAGA-3'. The following $\beta$-actin (the internal control) primers were used: sense primer, 5'-CTCCTCCTGAGCGCAAGT ACTC-3'; antisense primer, 5'-TCCTGCTTGCTGATCCA
CATC-3'. Real-time monitoring of PCR reactions was performed using the Light Cycler System and SYBR-Green I Master (Roche) according to the manufacturer's instructions. Quantitative real-time PCR was performed with the following cycling conditions; initial denaturation at $95^{\circ} \mathrm{C}$ for $10 \mathrm{~min}$, followed by 40 cycles of $95^{\circ} \mathrm{C}$ for $10 \mathrm{sec}$, annealing at $60^{\circ} \mathrm{C}$ for $10 \mathrm{sec}$, and extension at $72^{\circ} \mathrm{C}$ for $10 \mathrm{sec}$. After amplification, the relative expression level of $C D 47$ was obtained by dividing the amount of $C D 47$ mRNA by the amount of $\beta$-actin mRNA in each sample.

Re-analysis for GSE6188. The Gene Expression Omnibus public microarray database GSE6188 from the National Center for Biotechnical Information (21) was re-analyzed by Subio Platform Inc., Tokyo, Japan. Significantly different ( $\geq 2$-fold change) probes between cancerous tissues (153 samples) and normal tissues (104 samples) were extracted. The clinical status of the original tissue samples was determined in over half of the 48 donors with 2 cancerous tissues and 2 adjacent normal tissues.

Validation of miR-133a expression in clinical samples. cDNA for miR-133a quantitative real-time RT-PCR was synthesized from $10 \mathrm{ng}$ of total RNA using the TaqMan microRNA Reverse Transcription Kit and specific stem-loop reverse transcription primers (Applied Biosystems) according to the manufacturer's protocol. The $15 \mu \mathrm{l}$ reactions were incubated in a 96-well plate using the following temperature profile: $16^{\circ} \mathrm{C}$ for $30 \mathrm{~min}$ followed by $40^{\circ} \mathrm{C}$ for $30 \mathrm{~min}$ and $85^{\circ} \mathrm{C}$ for $5 \mathrm{~min}$. PCR was performed in a LightCycler ${ }^{\mathrm{TM}} 480$ System (Roche). The $20 \mu \mathrm{l}$ of PCR mix including the LightCycler 480 Probes Master kit (Roche) was incubated in a 96-well optical plate at $95^{\circ} \mathrm{C}$ for $10 \mathrm{~min}$ and then followed by 45 cycles of $95^{\circ} \mathrm{C}$ for $10 \mathrm{sec}$ and $60^{\circ} \mathrm{C}$ for $30 \mathrm{sec}$. The expression levels of miR-133a were normalized to that of the small nuclear RNA RNU6B and analyzed using the $2^{-\Delta \Delta \mathrm{Ct}}$ method.

ESCC cell line. The human ESCC cell line TE-8 was kindly provided by Dr T. Nishihira (Institute of Development, Aging and Cancer, Tohoku University School of Medicine, Sendai, Japan). TE-8 cells were maintained in Roswell Park Memorial Institute (RPMI-1640 medium) (Wako Pure Chemical Industries) supplemented with $10 \%$ fetal bovine serum and antibiotics (100 U/ml penicillin and $100 \mu \mathrm{g} / \mathrm{ml}$ streptomycin) and cultured in a humidified $5 \% \mathrm{CO}_{2}$ incubator at $37^{\circ} \mathrm{C}$.

Transfection of miR-133a precursor (Pre-miR-133a). Pre-miR miRNA Precursor Molecule mimicking miR-133a (Pre-miR133a; Applied Biosystems) or non-specific control miRNA (Pre-miR Negative Control \#1; Applied Biosystems) was transfected at $30 \mathrm{nmol} / 1$ into TE-8 cells using Lipofectamine RNAiMAX (Invitrogen) according to the manufacturer's instructions.

Plasmid construction. miR-133a target sequences in the 3'-UTR region of CD47 (Fig. 3A) were predicted using TargetScan (release 5.1: April 2009) and amplified from the genomic DNA of normal cells. The amplified fragment was inserted into the XhoI restriction sites of the dual-luciferase plasmid pmirGLO vector (Promega) using the In-Fusion ${ }^{\circledR}$ 

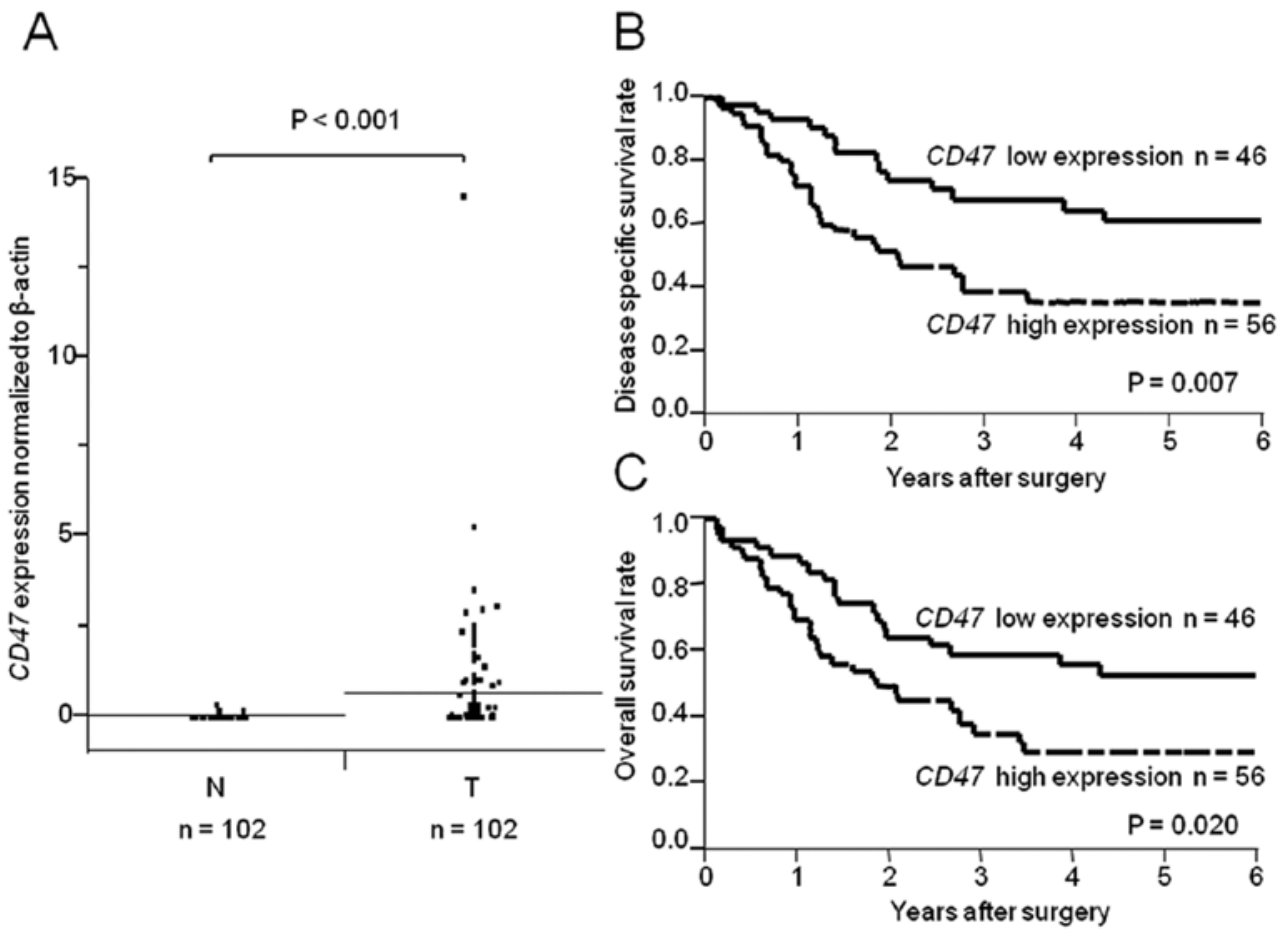

Figure 1. CD47 expression and prognosis in 102 ESCC cases. (A) CD47 levels assessed by real-time RT-PCR in cancerous (T) and non-cancerous (N) tissues from ESCC patients $(\mathrm{n}=102)$. CD47 levels in $\mathrm{T}$ were significantly higher than those in $\mathrm{N}(\mathrm{P}<0.001)$. Horizontal lines indicate each mean. (B and C) KaplanMeier curves based on $C D 47$ expression in ESCC. Disease-specific (5-year survival rates, high expression, 35.4\%; low expression, 60.8\%; $\mathrm{P}=0.007$ ) and overall survival rates (5-year survival rates: high expression, 29.4\%; low expression, 52.6\%; $\mathrm{P}=0.020$ ) of ESCC patients with high $C D 47$ expression were significantly lower than the rates among those with low $C D 47$ expression.

Dry-Down PCR Cloning kit (Clontech). Plasmid sequences were confirmed by sequencing using the following primers: sense, 5'-GCAAGATCGCCGTGTAATTCTAG-3'; anti-sense, 5'-AGAGGCCTCAGCAGGTCATA-3'.

Luciferase assay. TE-8 cells were seeded in a 96-well plate and co-transfected with $0.2 \mu \mathrm{g}$ of pmirGLO vecter, $100 \mathrm{nmol} / 1$ of Pre-miR-133a and $0.5 \mu \mathrm{l}$ of Lipofectamine RNAiMAX (Invitrogen) in $50 \mu \mathrm{l}$ of Opti-MEM Reduced-Serum Medium (Invitrogen). Pre-miR Negative Control \#1 was used as a control. Twenty-four hours following transfection, the activities of the firefly and Renilla luciferases in cell lysates were measured using the Dual-Glo ${ }^{\circledR}$ Luciferase Assay System (Promega) and the Fluoroskan Ascent FL (Thermo Fischer Scientific). Firefly luciferase activity was normalized to Renilla luciferase activity. All transfection experiments were conducted in triplicate.

Protein expression analysis. Western blot analysis was used to confirm the expression of CD47 in Pre-miR-133a transfected cells. Total protein was extracted from TE- 8 cells $48 \mathrm{~h}$ after transfection using PROPREP ${ }^{\text {тм }}$ protein extraction solution (Intron Biotechnology, Inc.). Total protein $(40 \mu \mathrm{g})$ was electrophoresed in an Any kD ${ }^{\mathrm{TM}}$ Mini-PROTEAN ${ }^{\circledR}$ TGX $^{\mathrm{TM}}$ Precast Gel (Bio-Rad Laboratories, Hercules, CA, USA) and then electrotransferred to a Hybond-enhanced nitrocellulose membrane (Amersham Pharmacia Biotech) at $200 \mathrm{~mA}$ for $180 \mathrm{~min}$ at $4^{\circ} \mathrm{C}$. The membrane was blocked with 5\% skim milk and CD47 protein was detected using CD47 mouse monoclonal antibody (Santa Cruz Biotechnology, Santa Cruz, CA) diluted 1:100. $\beta$-actin mouse monoclonal antibody (clone AC-74; Sigma) diluted 1:1000 served as a control. Bands on the membrane were detected using Retiga-4000R and QCapture Pro 6.0, an enhanced chemiluminescence detection system, according to the manufacturer's instructions (QImaging).

In vivo assay. Forty-eight hours following transfection with Pre-miR-133a or Pre-miR Negative Control \#1, 6-week-old female $\mathrm{BALB} / \mathrm{c}$ nude mice received subcutaneous injections of $4 \times 10^{6}$ of TE- 8 cells. The tumor volume was determined by caliper measurements at day 14 and calculated using the formula: Volume $=\mathrm{S} \times \mathrm{S} \times \mathrm{L} / 2$, where $\mathrm{S}$ is the short length of the tumor in $\mathrm{mm}$ and $\mathrm{L}$ is the long length of the tumor in $\mathrm{mm}$.

Statistical analysis. Differences between two groups were estimated using the Student's t-test, the $\chi^{2}$ test, and the repeatedmeasures ANOVA. Kaplan-Meier curves were generated for disease-specific and overall survival, and statistical significance was determined using the log-rank test. Univariate and multivariate survival analyses were carried out using the Cox proportional hazards regression model. A probability value of $<0.05$ was considered significant. All statistical analyses were performed using the JMP5.0 software (SAS Institute Inc.).

\section{Results}

Association between CD47 expression and clinicopathological findings in ESCC patients. CD47 expression was higher in a subset of ESCC samples compared with corresponding non-cancerous tissues (Fig. 1A; $\mathrm{P}<0.001$ ). We divided the 102 
Table I. Relationship between $C D 47$ expression and clinicopathological features.

\begin{tabular}{lccc}
\hline & \multicolumn{2}{c}{ CD47 expression, $\mathrm{n}$} & \\
\cline { 2 - 3 } & $\begin{array}{c}\text { Low } \\
\text { Factors }\end{array}$ & $\begin{array}{c}\text { High } \\
(\mathrm{n}=56)\end{array}$ & P-value \\
\hline Gender & & & \\
Male & 40 & 49 & 0.935 \\
Female & 6 & 7 & \\
Age (years) (mean \pm SD) & $65.0 \pm 8.0$ & $64.8 \pm 7.9$ & 0.864 \\
Tumor depth & & & \\
T1 & 12 & 7 & 0.248 \\
T2 & 7 & 6 & \\
T3 & 23 & 37 & \\
T4 & 4 & 6 & \\
Lymph node metastasis & & & \\
N0 & 20 & 14 & $0.049^{\mathrm{a}}$ \\
N1 & 26 & 42 & \\
Distant metastasis & & & \\
M0 & 41 & 46 & 0.316 \\
M1 & 5 & 10 & \\
Lymphatic invasion & & & \\
Negative & 40 & 54 & 0.074 \\
Positive & 6 & 2 & \\
Venous invasion & & & \\
Negative & 34 & 47 & 0.214 \\
Positive & 12 & 9 & \\
Tumor stage & & & \\
I & & & \\
II & 17 & 16 & 0.148 \\
III & 5 & 13 & \\
IV & & & \\
\hline
\end{tabular}

${ }^{\mathrm{a}} \mathrm{P}<0.05$.

ESCC patients into two groups according to the level of $C D 47$ expression in their cancerous tissue. The cut-off point was the median level of $C D 47$ expression in non-cancerous tissues. The correlation between $C D 47$ expression and the clinicopathologic characteristics of patients is shown in Table I. The $C D 47$ high expression group $(\mathrm{n}=56)$ showed more extensive lymph node metastasis than the low expression group $(n=46$; $\mathrm{P}=0.049$ ). However, no significant differences were observed regarding gender, age, tumor depth, distant metastasis, lymphatic invasion, venous invasion, or TNM stage.

Prognostic significance of CD47 expression in ESCC patients. The 5-year disease-specific survival rates of ESCC patients in the $C D 47$ high expression group were significantly lower than those in the low $C D 47$ expression group (high expression, 35.4\%; low expression, 60.8\%; $\mathrm{P}=0.007$; Fig. 1B). The 5-year overall survival rates of ESCC patients in the high expression group were also significantly lower than those in the low expression group (high expression, 29.4\%; low expression, 52.6\%; P=0.020; Fig. 1C). Univariate analysis of diseasespecific survival revealed that the relative level of tumor depth, lymph node metastasis, distant metastasis, lymphatic invasion, and CD47 expression were prognostic factors (Table II). Multivariate analysis of the five factors found to be significant in univariate analysis showed that high expression of $C D 47$ $(\mathrm{P}=0.045)$, tumor depth $(\mathrm{P}=0.042)$ and lymph node metastasis $(\mathrm{P}=0.019$; Table II) are independent prognostic factors of poor survival.

Re-analysis of GSE6188 and miR-133a expression in ESCC. In search for $C D 47$ regulatory miRNAs downregulated in ESCC, we found five downregulated miRNAs during re-analysis of a public microarray database (Fig. 2A). Among them, miR-133a expression was validated in 102 clinical samples of ESCC and adjacent non-cancerous tissues. The expression of miR-133a was significantly lower in cancerous tissues compared to noncancerous tissues $(\mathrm{P}<0.001$; Fig. 2B).

miR-133a directly binds the 3'-UTR of CD47 and regulates its protein expression in ESCC. MiR-133a was predicted to be an in silico regulator of $C D 47$ by TargetScan 5.0 (30) (Fig. 3A). We found an inverse correlation between miR-133a and CD47 expression in 102 clinical samples of ESCC. High levels of miR-133a were associated with low $C D 47$ expression $(\mathrm{P}<0.001 ;$ Fig. 3B). The luciferase reporter assay demonstrated that the luminescence intensity was significantly decreased in Pre-miR-133a transfectants (Fig. 3C; $\mathrm{P}<0.001$ ), suggesting that miR-133a has actual target sites in the 3'-UTR of CD47 mRNA in ESCC. The transient transfection of Pre-miR-133a also repressed CD47 protein levels in ESCC (Fig. 3D).

Tumor suppressive activity of miR-133a in vivo. A mouse xenograft model was used to evaluate the tumor suppressive activity of miR-133a (Fig. 4A). Pre-miR-133a strongly inhibited the tumorigenic potential of TE-8 cells in vivo (5 of 10; 50\%) compared with the negative control $(8$ of $8 ; 100 \%)(\mathrm{P}=0.036$, Fig. 4B). Moreover, Pre-miR-133a significantly inhibited tumor volume growth on Day 14 after injection ( $\mathrm{P}=0.007$; Fig. 4B).

\section{Discussion}

The expression level of miR-133a in primary ESCC was lower than in corresponding normal esophageal tissues, a finding which is consistent with the results of a previous microarray expression study (21). Because tumor suppressive miRNAs are usually underexpressed in tumors, it has been suggested that miR-133a functions as a tumor suppressor. Indeed, this study revealed that miR-133a inhibited tumor growth in an ESCC xenograft mouse model. Kano et al demonstrated that transfection of miR-133a inhibits cell proliferation and cell invasion in ESCC through the regulation of FSCN1, which is usually increased in tumors compared to normal epithelium (31). FSCN1 overexpression is significantly associated with poor prognosis in ESCC (32). These in vivo and in vitro findings suggest that miR-133a contributes to decreases in cell proliferation. Furthermore, our data revealed that miR-133a inhibits tumor xenograftment, suggesting that miR-133a could play a key role in tumorigenesis in ESCC. 
Table II. Results of univariate and multivariate analysis of clinicopathological factors affecting disease-specific survival rates following surgery.

\begin{tabular}{|c|c|c|c|c|c|c|}
\hline \multirow[b]{2}{*}{ Clinicopathological variable } & \multicolumn{3}{|c|}{ Univariate analysis } & \multicolumn{3}{|c|}{ Multivariate analysis } \\
\hline & RR & $95 \% \mathrm{CI}$ & P-value & $\mathrm{RR}$ & $95 \% \mathrm{CI}$ & P-value \\
\hline Gender (male/female) & 1.092 & $0.673-1.615$ & 0.671 & - & - & - \\
\hline Age $(<65 / \geq 65)$ & 1.065 & $0.7965-1.427$ & 0.671 & - & - & - \\
\hline Depth of tumor invasion $(\mathrm{T} 1 / \mathrm{T} 2,3,4)$ & 2.019 & $1.278-3.698$ & $0.001^{\mathrm{a}}$ & 1.665 & $1.018-3.143$ & $0.042^{\mathrm{a}}$ \\
\hline Lymph node metastasis (N0/N1) & 1.941 & $1.356-2.958$ & $<0.001^{\mathrm{a}}$ & 1.584 & $1.073-2.488$ & $0.019^{\mathrm{a}}$ \\
\hline Distant metastasis (M0/M1) & 1.662 & $1.153-2.304$ & $0.008^{\mathrm{a}}$ & 1.284 & $0.881-1.810$ & 0.185 \\
\hline Lymphatic invasion (negative/positive) & 2.354 & $1.098-9.913$ & $0.023^{\mathrm{a}}$ & 1.092 & $0.444-4.810$ & 0.870 \\
\hline Venous invasion (negative/positive) & 1.459 & $0.985-2.369$ & 0.060 & - & - & - \\
\hline miR-133a expression (high/low) & 1.117 & $0.835-1.499$ & 0.455 & - & - & - \\
\hline CD47 expression (low/high) & 1.515 & $1.118-2.097$ & $0.007^{\mathrm{a}}$ & 1.371 & $1.007-1.908$ & $0.045^{\mathrm{a}}$ \\
\hline
\end{tabular}

RR, relative risk; CI, confidence interval; ${ }^{\mathrm{P}}<0.05$.
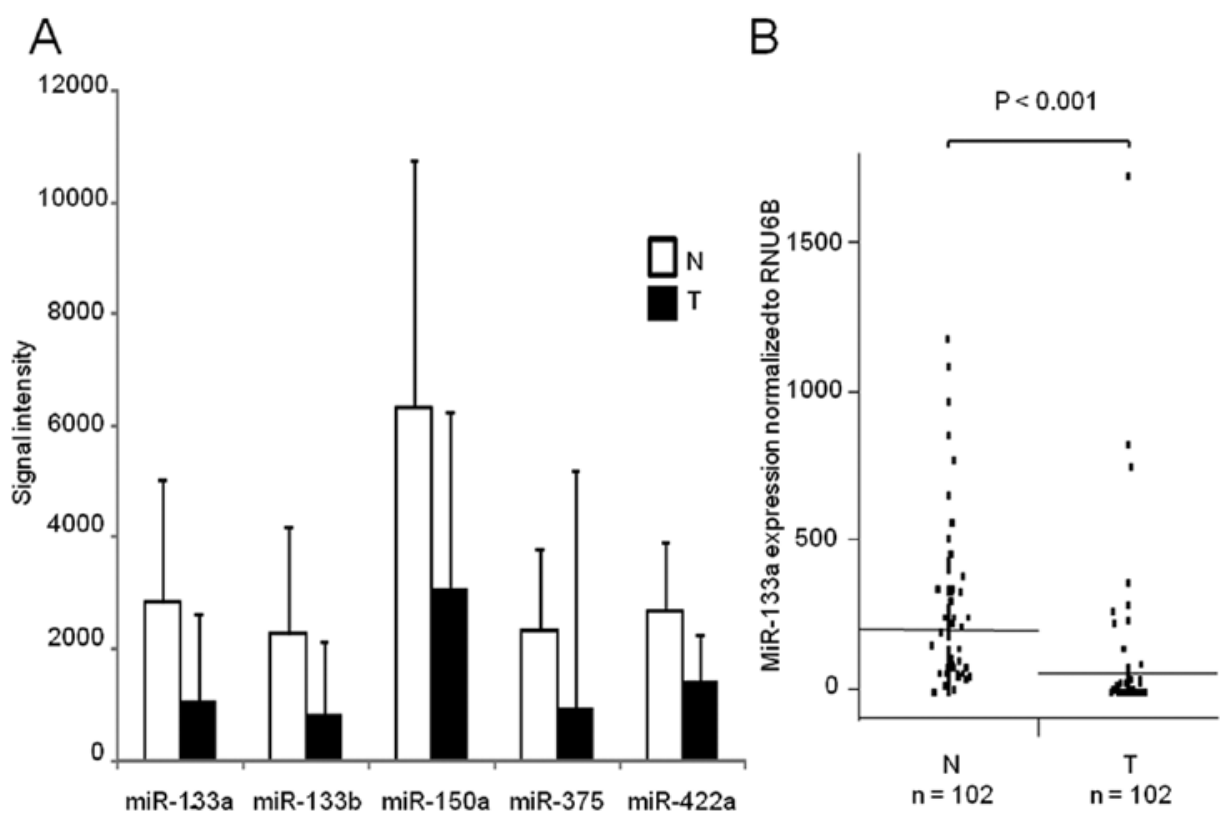

Figure 2. Re-analysis of a microarray database and miR-133a expression in 102 ESCC cases. (A) Five miRNAs downregulated in ESCC were found after re-analysis of the public microarray database GSE6188. The fold changes [cancerous tissues (T)/non-cancerous tissues (N)] were miR-133a, 0.366; miR-133b, 0.350; miR-150, 0.482; miR-375, 0.409; and miR-422a, 0.529. The error bar represents the mean \pm SD. (B) miR-133a expression in T and N from ESCC patients $(n=102)$ by real-time RT-PCR. miR-133a levels in $\mathrm{T}$ were significantly lower than those in $\mathrm{N}(\mathrm{P}<0.001)$. Horizontal lines indicate each mean.

Luciferase assays supported the premise of a specific interaction between miR-133a and the 3'-UTR of $C D 47$, a protein that inhibits phagocytosis. The negative modulatory effect of miR-133a was substantiated further by a strong inverse relationship between the expression levels of $C D 47$ mRNA and miR-133a. The significant reduction of $C D 47$ protein in an ESCC cell line following Pre-miR-133a transfection suggests that miR-133a directly regulates $C D 47$ expression. In contrast, it has been demonstrated that hematopoietic cells from $C D 47$ knockout mice were rapidly cleared from the bloodstream by macrophages in syngeneic wild-type mice $(7,33)$. Chao et al also reported that monoclonal antibody against $C D 47$ enabled phagocytosis of acute lymphoblastic leukemia cells by macrophages in vitro and inhibited tumor engraftment in vivo (13). These findings suggest that the rapid rejection of xenograft cells by recipient macrophages could have resulted from the critical role of $C D 47$ in self protection. These studies support the idea that miR-133a contributes to anti-tumorigenesis in ESCC through the regulation of $C D 47$ expression. To the best of our knowledge, this is the first study describing the clinical significance of $C D 47$ and its regulation by miR-133a in ESCC.

The expression of $C D 47$ is significantly correlated with lymph node metastasis, the main cause of death in most cancer patients. In particular, lymph node metastasis leads to poor prognosis after surgical resection in ESCC patients (34). 
A

Position 1623-1629 of CD473' UTR 5'-UUUCAUGUUG----GGACCAAG-3'

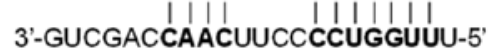

Hsa-miR-133a

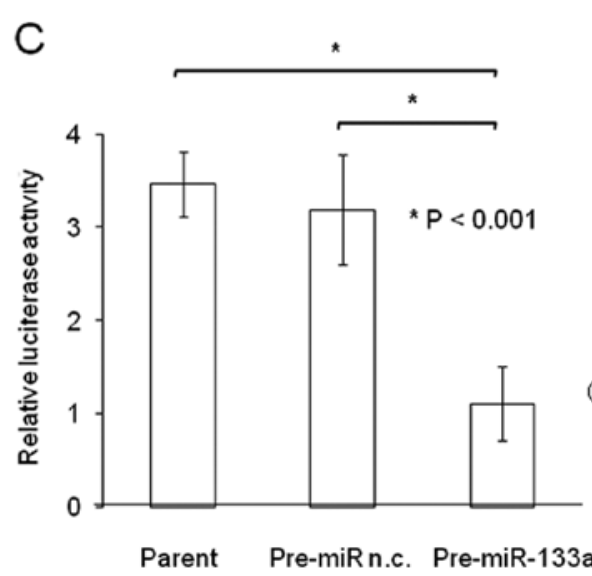

B

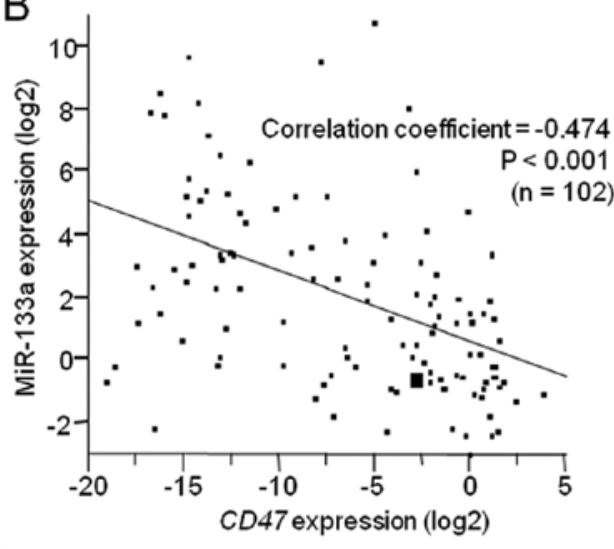

D

CD47

$(47-52 \mathrm{kDa})$

$\beta$-actin

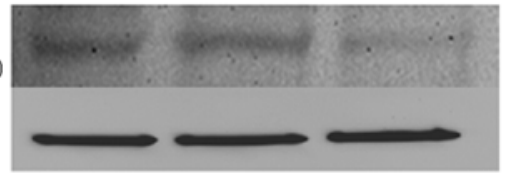

Parent Pre-miR n.c. Pre-miR-133a

Figure 3. Regulation of $C D 47$ expression by miR-133a. (A) The miR-133a-binding site in the $C D 47$ 3'-UTR. Putative conserved target sites in the $C D 47$ 3'-UTR were identified with the TargetScan database. (B) Inverse correlation between miR-133a and CD47 expression in ESCC. High levels of miR-133a were associated with low $C D 47$ expression $(\mathrm{P}<0.001)$. miR-133a and $C D 47$ expression were examined by real-time PCR analysis and normalized to RNU6B and $\beta$-actin, respectively. (C) Luciferase assay in TE-8 cells. miR-133a transfectants showed lower luciferase activity than control and parent TE-8 cells ("P $<0.001$ ). The error bar represents the SD from 8 replicates. Left, $C D 47-3$ '-UTR luciferase vector only; middle, $C D 47-3$ '-UTR luciferase vector +Pre-miR n.c.; right, CD47-3'-UTR luciferase vector +Pre-miR-133a. (D) Western blots indicated that $C D 47$ protein expression is decreased by the ectopic expression of miR-133a. Left, parent; middle, Pre-miR n.c.; right, Pre-miR-133a.

A

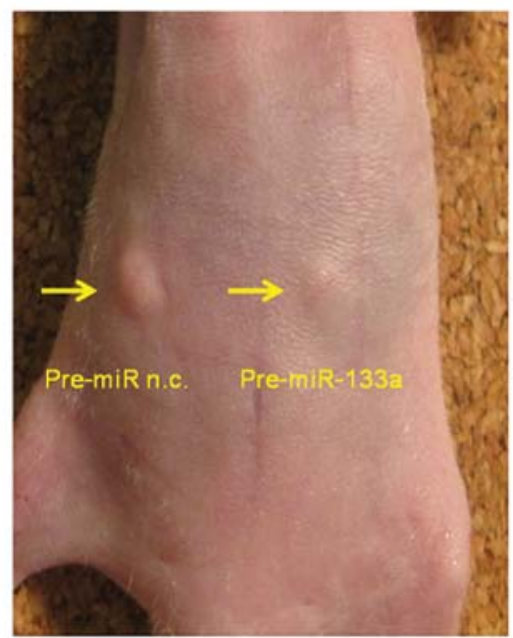

B

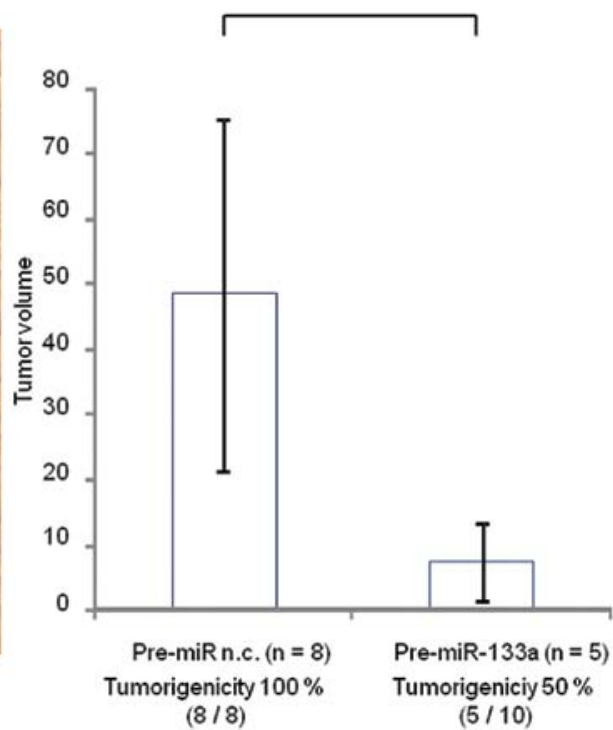

Figure 4. miR-133a inhibits tumorigenicity and tumor growth in vivo. (A) TE-8 cells transfected with Pre-miR-133a or Pre-miR negative control (n.c.) were injected subcutaneously into female BALB/c nude mice. The representative image was taken on Day 7. Left, Pre-miR n.c. transfectant; right, Pre-miR-133a transfectant. (B) Comparison of tumor size in nude mouse transplanted with TE-8 cells transfected with Pre-miR-133a or Pre-miR n.c. 14 days after subcutaneous injection. The incidence of tumors (tumorigenicity) is shown below. The bar represents the mean \pm SD.

However, tumor immunosurveillance is a well-established mechanism for the regulation of tumor progression (11), and macrophages in the lymph node sinus are the front-line endogenic defense against metastasis (35). Asano et al showed that macrophages phagocytose dead tumor cells transported via lymphatic flow and subsequently cross-present tumor antigens to $\mathrm{CD}^{+} \mathrm{T}$ cells, resulting in activation of the antitumor immunity system (36). Therefore, ESCC characterized by high CD47 expression may have the ability to evade phagocytosis, and after acquiring invasiveness, to participate 
in the promotion of lymph node metastasis. Multivariate Cox proportional hazard regression analysis revealed that CD47 expression had a significantly worse prognostic impact $(\mathrm{P}=0.045)$ on survival of ESCC patients independent of tumor depth $(\mathrm{P}=0.042)$ and lymph node metastasis $(\mathrm{P}=0.019)$. Novel biomarkers that are capable of precisely identifying high risk ESCC patients may provide the tools for determining appropriate therapeutic strategies. Our data indicate that $C D 47$ could be of value as a novel prognostic marker for the identification of aggressive ESCC.

Although miRNAs show signs of being a new class of targets for therapeutic applications (37), the development of effective and safe approaches for sequence-specific antagonism of miRNAs in vivo remains a significant scientific and therapeutic challenge $(38,39)$. On another front, $C D 47$ was identified as a therapeutic antibody target in acute myeloid leukemia, non-Hodgkin's lymphoma, bladder cancer and acute lymphoblastic leukemia $(12,13,40-42)$. However, until now, the regulation of $C D 47$ expression is not well understood. The identification of miR133a-mediated $C D 47$ regulation might provide a promising new therapeutic strategy in treating ESCC.

In conclusion, our data indicate that $C D 47$ is an independent prognostic marker that is regulated by miR-133a, which may function as a tumor suppressor in ESCC. This correlation could provide new insight into the mechanism of cancer progression and a promising candidate for a novel therapeutic target in ESCC.

\section{Acknowledgements}

We thank Ms. Masako Shin for her excellent technical assistance.

\section{References}

1. Enzinger PC and Mayer RJ: Esophageal cancer. N Engl J Med 349: 2241-2252, 2003.

2. Parkin DM, Bray F, Ferlay J and Pisani P: Global cancer statistics, 2002. CA Cancer J Clin 55: 74-108, 2005.

3. Malthaner RA, Wong RK, Rumble RB and Zuraw L: Neoadjuvant or adjuvant therapy for resectable esophageal cancer: a systematic review and meta-analysis. BMC Med 2: 35, 2004.

4. Gebski V, Burmeister B, Smithers BM, Foo K, Zalcberg J and Simes J: Survival benefits from neoadjuvant chemoradiotherapy or chemotherapy in oesophageal carcinoma: a meta-analysis. Lancet Oncol 8: 226-234, 2007.

5. Tepper J, Krasna MJ, Niedzwiecki D, et al: Phase III trial of trimodality therapy with cisplatin, fluorouracil, radiotherapy, and surgery compared with surgery alone for esophageal cancer: CALGB 9781. J Clin Oncol 26: 1086-1092, 2008.

6. Shinohara M, Ohyama N, Murata Y, et al: CD47 regulation of epithelial cell spreading and migration, and its signal transduction. Cancer Sci 97: 889-895, 2006.

7. Oldenborg PA, Zheleznyak A, Fang YF, Lagenaur CF, Gresham HD and Lindberg FP: Role of CD47 as a marker of self on red blood cells. Science 288: 2051-2054, 2000.

8. Okazawa H, Motegi S, Ohyama N, et al: Negative regulation of phagocytosis in macrophages by the CD47-SHPS-1 system. J Immunol 174: 2004-2011, 2005.

9. van den Berg TK and van der Schoot CE: Innate immune 'self' recognition: a role for CD47-SIRPalpha interactions in hematopoietic stem cell transplantation. Trends Immunol 29: 203-206, 2008.

10. Oldenborg PA, Gresham HD, Chen Y, Izui S and Lindberg FP: Lethal autoimmune hemolytic anemia in CD47-deficient nonobese diabetic (NOD) mice. Blood 99: 3500-3504, 2002.
11. Jaiswal S, Chao MP, Majeti R and Weissman IL: Macrophages as mediators of tumor immunosurveillance. Trends Immunol 31: 212-219, 2010.

12. Majeti R, Chao MP, Alizadeh AA, et al: CD47 is an adverse prognostic factor and therapeutic antibody target on human acute myeloid leukemia stem cells. Cell 138: 286-299, 2009.

13. Chao MP, Alizadeh AA, Tang C, et al: Therapeutic antibody targeting of CD47 eliminates human acute lymphoblastic leukemia. Cancer Res 71: 1374-1384, 2011.

14. Nagahara M, Mimori K, Kataoka A, et al: Correlated expression of CD47 and SIRPA in bone marrow and in peripheral blood predicts recurrence in breast cancer patients. Clin Cancer Res 16: 4625-4635, 2010

15. Junker A, Krumbholz M, Eisele S, et al: MicroRNA profiling of multiple sclerosis lesions identifies modulators of the regulatory protein CD47. Brain 132: 3342-3352, 2009.

16. Valencia-Sanchez MA, Liu J, Hannon GJ and Parker R: Control of translation and mRNA degradation by miRNAs and siRNAs. Genes Dev 20: 515-524, 2006.

17. Bartel DP: MicroRNAs: genomics, biogenesis, mechanism, and function. Cell 116: 281-297, 2004.

18. Bartel DP: MicroRNAs: target recognition and regulatory functions. Cell 136: 215-233, 2009.

19. Lu J, Getz G, Miska EA, et al: MicroRNA expression profiles classify human cancers. Nature 435: 834-838, 2005.

20. Volinia S, Calin GA, Liu CG, et al: A microRNA expression signature of human solid tumors defines cancer gene targets. Proc Natl Acad Sci USA 103: 2257-2261, 2006.

21. Guo Y, Chen Z, Zhang L, et al: Distinctive microRNA profiles relating to patient survival in esophageal squamous cell carcinoma. Cancer Res 68: 26-33, 2008.

22. Childs G, Fazzari M, Kung G, et al: Low-level expression of microRNAs let-7d and miR-205 are prognostic markers of head and neck squamous cell carcinoma. Am J Pathol 174: 736-745, 2009.

23. Wong TS, Liu XB, Wong BY, Ng RW, Yuen AP and Wei WI: Mature miR-184 as potential oncogenic microRNA of squamous cell carcinoma of tongue. Clin Cancer Res 14: 2588-2592, 2008

24. Nohata N, Hanazawa T, Kikkawa N, et al: Caveolin-1 mediates tumor cell migration and invasion and its regulation by miR-133a in head and neck squamous cell carcinoma. Int J Oncol 38: 209-217, 2011.

25. Chiyomaru T, Enokida H, Tatarano S, et al: miR-145 and miR-133a function as tumour suppressors and directly regulate FSCN1 expression in bladder cancer. Br J Cancer 102: 883-891, 2010.

26. Song T, Xia W, Shao N, et al: Differential miRNA expression profiles in bladder urothelial carcinomas. Asian Pac J Cancer Prev 11: 905-911, 2010.

27. Bandres E, Cubedo E, Agirre X, et al: Identification by real-time PCR of 13 mature microRNAs differentially expressed in colorectal cancer and non-tumoral tissues. Mol Cancer 5: 29, 2006.

28. Missiaglia E, Shepherd CJ, Patel S, et al: MicroRNA-206 expression levels correlate with clinical behaviour of rhabdomyosarcomas. Br J Cancer 102: 1769-1777, 2010.

29. Rao PK, Missiaglia E, Shields L, et al: Distinct roles for miR-1 and miR-133a in the proliferation and differentiation of rhabdomyosarcoma cells. FASEB J 24: 3427-3437, 2010.

30. Lewis BP, Burge CB and Bartel DP: Conserved seed pairing, often flanked by adenosines, indicates that thousands of human genes are microRNA targets. Cell 120: 15-20, 2005.

31. Kano M, Seki N, Kikkawa N, et al: miR-145, miR-133a and miR-133b: Tumor suppressive miRNAs target FSCN1 in esophageal squamous cell carcinoma. Int J Cancer 127: 2804-2814, 2010.

32. Hashimoto $\mathrm{Y}$, Ito $\mathrm{T}$, Inoue $\mathrm{H}$, et al: Prognostic significance of fascin overexpression in human esophageal squamous cell carcinoma. Clin Cancer Res 11: 2597-2605, 2005.

33. Wang H, Madariaga ML, Wang S, Van Rooijen N, Oldenborg PA and Yang YG: Lack of CD47 on non-hematopoietic cells induces split macrophage tolerance to CD47null cells. Proc Natl Acad Sci USA 104: 13744-13749, 2007.

34. Kuwano H, Nakajima M, Miyazaki $\mathrm{T}$ and Kato H: Distinctive clinicopathological characteristics in esophageal squamous cell carcinoma. Ann Thorac Cardiovasc Surg 9: 6-13, 2003.

35. Nagata $H$, Arai T, Soejima Y, Suzuki H, Ishii $H$ and Hibi $T$ : Limited capability of regional lymph nodes to eradicate metastatic cancer cells. Cancer Res 64: 8239-8248, 2004. 
36. Asano K, Nabeyama A, Miyake Y, et al: CD169-positive macrophages dominate antitumor immunity by crosspresenting dead cell-associated antigens. Immunity 34: 85-95, 2011.

37. Soifer HS, Rossi JJ and Saetrom P: MicroRNAs in disease and potential therapeutic applications. Mol Ther 15: 2070-2079, 2007.

38. Elmen J, Lindow M, Schutz S, Lawrence M, et al: LNA-mediated microRNA silencing in non-human primates. Nature 452 : 896-899, 2008

39. Silvestri P, Di Russo C, Rigattieri S, et al: MicroRNAs and ischemic heart disease: towards a better comprehension of pathogenesis, new diagnostic tools and new therapeutic targets. Recent Pat Cardiovasc Drug Discov 4: 109-118, 2009.
40. Chan KS, Espinosa I, Chao M, et al: Identification, molecular characterization, clinical prognosis, and therapeutic targeting of human bladder tumor-initiating cells. Proc Natl Acad Sci USA 106: 14016-14021, 2009

41. Chao MP, Alizadeh AA, Tang C, et al: Anti-CD47 antibody synergizes with rituximab to promote phagocytosis and eradicate non-Hodgkin lymphoma. Cell 142: 699-713, 2010.

42. Chao MP, Alizadeh AA, Tang C, et al: Therapeutic antibody targeting of CD47 eliminates human acute lymphoblastic leukemia. Cancer Res 71: 1374-1384, 2010. 